\title{
Summary of conference $\left(40^{\prime}+5^{\prime}\right)$
}

\section{Paolo Gondolo*}

University of Utah, USA

E-mail: paolo@physics.utah.edu

There is now overwhelming cosmological evidence for non-baryonic dark matter that is not made of any known elementary particle. Physicists and astronomers are in the exploratory stages to figure out its nature, with many ideas currently explored by both theorists and experimentalists. Nature is teasing them with anomalous signals that may reveal the identity of dark matter.

Identification of dark matter 2008

August 18-22, 2008

Stockholm, Sweden

${ }^{*}$ Speaker. 
The biennial conferences on the Identification of Dark Matter focus on one of the major question of physics and astronomy: the nature of the dark matter that makes up about $85 \%$ of the mass in the Universe. The main focus of the meeting was on searches for non-baryonic cold dark matter, but brief reviews of cosmology were also presented, as well as some of the experimental efforts to measure the neutrino mass with neutrinoless double-beta decay.

Current cosmological data are compatible with a simple model of the Universe made of five components: ordinary matter (baryons), electromagnetic radiation, neutrinos, cold dark matter and a cosmological constant (dark energy). A recent analysis of their mass and energy densities [1], based on the combination of cosmic microwave background fluctuations, high-redshift supernovas, and baryon acoustic oscillations, finds the following values and constraints: ${ }^{1}$

$$
\begin{gathered}
\rho_{\mathrm{b}}=(0.4239 \pm 0.011) \mathrm{yg} / \mathrm{m}^{3}, \quad \rho_{\mathrm{r}}=0.000464 \mathrm{yg} / \mathrm{m}^{3}, \quad \rho_{v} \leq 0.13 \mathrm{yg} / \mathrm{m}^{3}(95 \% \mathrm{CL}) \\
\rho_{\mathrm{cdm}}=(2.124 \pm 0.064) \mathrm{yg} / \mathrm{m}^{3}, \quad \rho_{\Lambda}=(6.78 \pm 0.14) \mathrm{yg} / \mathrm{m}^{3} .
\end{gathered}
$$

Riess $^{2}$ spoke about the current strategies to measure the properties of dark energy, showing the many ways of attacking the problem as spokes on a "dark energy wheel:" determining the Hubble constant, searching for type Ia supernovas at low and high redshift, extending the large-scale structure surveys, improving the data on baryon acoustic oscillations, studying galaxy clusters through $\mathrm{X}$-rays and the Sunyaev-Zeldovich effect, examining correlations in and between the Cosmic Microwave Background and large-scale structure. Linder overviewed some of the current models for dark energy. Tegmark conveyed the high expectations to detect the $21-\mathrm{cm}$ radiation from hydrogen at high-redshift and thus for the first time observe the universe 'half-way' to the surface of last scattering.

Neutrinos may be a significant component of the Universe. Now that the masses of standard model neutrinos are known to be smaller than $1 \mathrm{eV}$ or so, neutrinos are recognized as constituents of hot dark matter. They are expected to be present today in the form of a Cosmic Neutrino Background, analogous to the Cosmic Microwave Background. They have been absent from the conference, perhaps because no suitable detector is under construction for them. The issue of neutrino masses was instead reviewed and some presentations focused on experimental efforts to measure the neutrino mass in neutrinoless double-beta decay.

Schwetz-Mangold gave a very nice review of the current situation in the measurement of neutrino masses and in the understanding of the mixing angles in the neutrino sector. The best graphical evidence for neutrino oscillations is the KamLAND plot showing the disappearance of electron antineutrinos as a function of the distance from the nuclear reactors in which they are produced. Flavor oscillations have been observed for reactor antineutrinos, neutrinos from the Sun, and atmospheric neutrinos. They have allowed the determination of the differences of the square of the neutrino masses $\Delta m^{2}$ between the lightest and the intermediate, and between the intermediate and the heaviest neutrinos. The remaining mass-squared difference, between the lightest and the heaviest, is still to be determined: if it is positive, the two lightest neutrinos are close in mass and the heaviest one is more separated (normal mass hierarchy); if it is negative, it is the two heavi-

\footnotetext{
${ }^{1}$ Densities are expressed in units of physical mass density through $E=m c^{2} ; 1 \mathrm{yg}=1$ yoctogram $=10^{-24} \mathrm{~g} ; \Omega h^{2}=$ $\rho /(18.78 \mathrm{yg})$; the error on $\rho_{\Lambda}$ is only an estimate based on the published error on $\Omega_{\Lambda}$.

${ }^{2}$ Author's names without an explicit reference number refer to contributions to These Proceedings.
} 
est neutrinos which are close in mass and the lightest one is separated (inverted mass hierarchy). Oscillations by themselves cannot determine the absolute mass of the neutrinos, however, nor can they determine if the neutrinos are Majorana or Dirac (i.e. if antineutrinos are or are not the same as neutrinos). Cosmology provides a link to the absolute mass of the neutrinos: since the mass differences from oscillations are no more than $\sim 0.1 \mathrm{eV}$, and experiments using the end-point of the Helium- 4 decay spectrum place an upper limit of $\sim 2 \mathrm{eV}$ on the mass of the neutrino, we know that all Standard Model neutrinos are light and thus behave like hot dark matter in cosmology. Hot dark matter tends to erase density fluctuations on small scales, but the detection of these fluctuations on the line of sight to quasars by means of the Lyman- $\alpha$ forest places an upper bound on the density of hot dark matter neutrinos. This is in essence the origin of the bound quoted in Eq. (1) above. It translates to an upper limit on the sum of the neutrino masses of $\sim 0.7 \mathrm{eV}$. If neutrinos are Majorana, the neutrino mass could be measured in the laboratory using the still hypothetical phenomenon of neutrinoless double-beta decay. Huge detectors have been constructed for this purpose, some of which were described at the conference (GERDA by Majorovitz, CUORE by Giuliani, KATRIN by Weinheimer), with amazing images of installing a giant metallic bottle or transporting a mammoth vessel through narrow streets of a village. The hopes are high that these efforts will produce a long sought-after measurement of the absolute neutrino mass.

Turning to cold dark matter, the main topic of the conference, I wish to first point out two pieces of evidence that show what cold dark matter is not. The first is the match between the observed and theoretical matter power spectrum around the wave number corresponding to the time of matter-radiation equality (when the energy density of radiation, which scales as volume ${ }^{-4 / 3}$, equaled the energy density of matter, which scales as volume ${ }^{-1}$ ). At that time, density fluctuations in the matter started to grow, and modify the power spectrum at larger wave numbers (i.e., scales smaller than the horizon at matter-radiation equality). In the absence of dark matter, the spectrum would show oscillations in wave number, corresponding to the acoustic oscillations of the baryons. Under the dominance of dark matter (defined here as something that interacts only weakly with the plasma), the spectrum would be basically smooth (with only small ripples to provide a telltale sign of the baryon acoustic oscillations). The latter is what is observed [2,3]. Thus dark matter interacts very weakly, if at all, with the baryon-photon plasma. This is one of the best pieces of evidence for dark matter that is non-baryonic.

The second constraint on the nature of cold dark matter that I want to mention is that it does not form objects of the size and mass of planets. This kind of objects would have been detected in the halo of our Galaxy when they would pass in front of a background star and magnify its light (gravitational microlensing). As reported by Moniez, the lack of gravitational microlensing events with the expected properties in the EROS and OGLE data places an upper limit of about 5-10\% on the fraction of the Galactic dark halo in planet-like objects of mass between $10^{-7}$ and a few solar masses. Subhalos of similar masses made of elementary particles avoid such limits by having a much larger spatial extension.

The question of the nature of non-baryonic dark matter remains wide open. Several speakers presented theoretical ideas for new elementary particles as dark matter. There were presentations within the established patterns of axions, supersymmetry and extra dimensions (Bazzocchi, Beckwith, Gomez, Gurwich, Kim, Santoso, and Solberg) and also talks introducing fresh ideas that go beyond the established patterns, all inspired by anomalous signals (Cirelli, Finkbeiner, Hooper, 
Kouvaris, Weiner).

Detection of dark matter particles remains the ultimate goal, and high are the interest and excitement in searching for particle dark matter with any means possible: from producing it at particle accelerators to detecting it directly to observing it indirectly through its astrophysical effects. Progress in direct and indirect searches has been characterized by how much the unwanted background is reduced. In direct searches this has been accomplished through signal-to-noise discrimination and by the use of characteristic feature like the annual modulation. In indirect searches, one uses models of the background (which is often not fully represented) and features in the expected signals (in neutrinos, gamma-rays, positrons, antiprotons, etc.), such as their spectral shape or their angular distribution in the sky.

Accelerator searches at the Large Hadron Collider (which is planned to start science operations in 2009) have the potential to discover charged or colored particles connected to new physics, like supersymmetry or extra dimensions. The existence of associated neutral particles that could be the dark matter will have to be inferred either through missing energy measurements or theoretical considerations. That any discovered neutral particle is indeed the dark matter will ultimately have to be confirmed by astrophysical experiments. Gjelsten, of the ATLAS Collaboration, presented a thorough description of how supersymmetric particles will be searched for at the LHC, and drew the connection to supersymmetric dark matter. The LHC-dark matter connection was further explored in talks by Dutta, Fornasa, and Arrenberg, who stressed either the likely difficulty of measuring the dark matter particle mass at the LHC or the complementarity of LHC and dark matter searches in covering theoretical models.

Direct searches for particle dark matter have seen substantial progress during the past year, and this was reflected in the many recent results presented at the conference. Axion limits are steadily being improved and reach into the theoretically preferred region: van Bibber presented the first results from the Axion Dark Matter eXperiment (ADMX), Steffen presented the constraints from the GammeV experiment, Lakic presented the search for solar axions with CAST, and Inoue presented the Tokyo axion helioscope experiment.

The lion's share of the presentations was on searches for weakly-interacting massive particles (WIMPs). The DAMA collaboration (Belli) confirmed their previous detection of an annual modulation in the rate of energy deposited into their sodium iodide detectors. No other explanation is currently available for the observed modulation but dark matter particles. However, other detectors using different materials and having comparable sensitivities do not report dark matter events. The strongest current limits are from the XENON10 experiment (Aprile) at high particle masses and the CDMS (Hall), COUPP (Cooper) and TEXONO (Wong) experiments at low masses. WIMP interpretations of the DAMA modulation compatible with the other experiments have been known to be possible at relatively low WIMP masses (around $10 \mathrm{GeV}$ or so). One of the non-traditional interpretations, namely inelastic dark matter, was presented by Weiner.

Much progress is foreseen in the coming years. Reports were presented on several ongoing or upcoming direct detection experiments: XENON100 (Aprile, Baudis), LUX (Gaitskell, McKinsey), PICASSO (Davour), XMASS (Suzuki), WArP (Fiorillo), ULTIMA (Bunkov), DEAP/CLEAN (Boulay), EURECA (Kraus), EDELWEISS (Defay), CRESST (Petricca), ZEPLIN-III (Sumner), and ROSEBUD (Ortigoza-Paredes). Background studies and new detector ideas were presented jointly with a meeting of the Integrated Large Infrastructures for Astroparticle Science (ILIAS) 
European project (Spooner, Kudryavtsev, Golwala, Selvi, Gomez Maluenda, Tomasello, Kozlov, Nones, see also Nygren). Analyses of bounds and possible mass measurements were presented by Green and Shan. Finally, substantial progress was reported in the development of a new generation of direction-sensitive devices that will be able to determine the direction from which WIMPs would be coming from: DRIFT (Spooner), DM-TPC (Sciolla), NEWAGE (Nishimura), NIT (Naka), and MIMAC (Santos). Directional detection will not only facilitate background discrimination, but will also allow the measurement of the local phase space distribution of dark matter particles. These detectors will usher in an era of dark matter astronomy.

Indirect searches for particle dark matter have brought us new puzzles and new ideas. Indirect signals include high-energy neutrinos, gamma-rays, antiprotons, and positrons from particle dark matter, and more generally the effects of particle dark matter on astrophysical objects. Since the rate of dark matter annihilation scales as the square of the dark matter density, annihilation of WIMPs is important whenever the dark matter density is high: in the Sun and Earth, in the Milky Way halo and the Galactic Center, in external galaxies, and, as recently stressed, in stars living in dense dark matter environments.

The challenge with indirect detection methods is understanding the background to such a level that one is confident about the detection of a dark matter signal. The still imperfect understanding of cosmic ray propagation in the galaxy was brought back to mind by the talks of Morselli and Gebauer. The unprecedented quality of the gamma-ray and cosmic-ray data that are now being collected will help solve these theoretical difficulties and will uncover a new view of the highenergy galaxy.

One of the highlights of the conference was the presentation of the preliminary measurement of the positron-to-electron ratio in cosmic rays by the PAMELA collaboration (Boezio). This measurement confirmed and significantly improved the detection of an anomalous rise at energies above $10 \mathrm{GeV}$ first observed by the HEAT detector a few years ago with much lower statistics. Morselli, while reviewing the status of theoretical models of cosmic ray propagation, mentioned astrophysical attempts at reproducing the observed excess. Theoretical papers attributing the positron excess to dark matter annihilation were quick to appear (even during the conference itself, see Cirelli's talk). However, a full understanding of the origin of the positron excess will have to await a better modeling of cosmic ray production and propagation in our galaxy. In this regard, the data that are coming in from the recently-launched Fermi Gamma-Ray Space Telescope (formerly GLAST) are of great importance.

The Fermi Telescope has also the tantalizing opportunity of discovering the annihilation of dark matter into high-energy gamma-rays, and in particular into mono-energetic gamma-rays. The prospects of detecting dark matter annihilation with Fermi were described in numerous contributions (Coehn-Tanugi, Sivertsson, Ylinen, Nuss, Vitale, Cuoco). Bringmann stressed the importance of radiative corrections in predicting the spectral shape of gamma-rays from WIMP annihilation. Kuhlen and Frenk held differing views about the expected sky distribution of gamma-rays from dark matter annihilation, both based on N-body simulations of the formation of our galaxy: Kuhlen claimed that bright gamma-ray spots from many small dark matter concentrations would be apparent, while Frenk claimed that a smooth increase of the gamma-ray intensity toward the Galactic Center would dominate the observations.

Other N-body simulations of the small-scale structure of the Galactic dark halo were presented 
by Vogelsberger, who discussed a new technique to study dark matter caustics, and by Read and Bruch, who showed the theoretical existence of a dark matter disk accompanying the visible one and discussed its consequences for current and future dark matter experiments. Dokuchaev expounded a criticism of the current methods to treat small dark subhalos, and Taylor alluded to the main points of controversy between theory and observation of halo substructure. On the observational side, Strigari reviewed the properties of the many newly discovered low-luminosity stellar systems in the Galactic halo, Host discussed the measurements of velocity anisotropy profiles in galaxy clusters, and Zackrisson summarized the current knowledge on the clustering of dark baryons (which are the baryons at low redshift that have still been unaccounted for). Keeton, Riehm, and Liesenborgs pointed out the use of gravitational lensing to search and detect small-scale dark matter structures.

The Galactic Center as an observation target to search for dark matter signals was the topic of several presentations. In recent years, it has become clear that for this purpose one needs to overcome the numerous astrophysical sources already detected in that direction. The topography of the Galactic Center region were reviewed by Eckart, together with the status and the expectations of multi-wavelength surveys. Regis reflected on the benefit of multi-wavelength observations for dark matter searches, while Hooper and Dobler focused on an excess in the microwave band as a possible signal from dark matter. Vitale spoke about the prospects of detecting a dark matter signal with the Fermi Telescope.

Gamma-ray telescopes on the ground are in steady progress. The status of very-high-energy gamma-ray astronomy was reviewed by Buckley, who indicated that current instruments (HESS, VERITAS, and MAGIC) have similar collection areas and have peak energy around 100-300 GeV. Biland and Farnier presented prospects for indirect dark matter searches with MAGIC and HESS, respectively.

Current limits on neutrino fluxes from dark matter annihilation in the Sun and Earth were displayed by de Clercq on behalf of the IceCUBE collaboration. The status of the ANTARES neutrino telescope, and preliminary analysis of their neutrino events, were presented by Ernenwein, who also discussed the sensitivity of ANTARES to neutrinos from supersymmetric dark matter. Coyle summarized the potential of KM3NET, a future kilometer-cubed neutrino telescope, for the indirect detection of dark matter. On the theoretical side, Blennow considered detailed calculations of neutrino propagation in the Sun and the Earth, and Peter how the dynamics of WIMPs in the Solar System affects the neutrino (and the direct detection) signals.

New theoretical studies were presented on the possibility of indirectly observing particle dark matter through its effects on stars. Scott presented the impact of dark matter annihilation upon the evolution of main-sequence stars and stars near black-holes. Gondolo and Spolyar presented the idea that the first stars to form in the universe may be "dark stars" powered by dark matter annihilation rather than nuclear fusion. Further elaborations on the topic were discussed by Ripamonti and Taoso.

The conference was rounded out by talks on antiprotons: PAMELA measurements were presented by Boezio, and future prospects for dark matter detection with PEBS were discussed by Gast. In addition, Edsjo described the DarkSUSY software for particle dark matter, and Rakic dissected a general-relativistic attempt to explain galactic rotation curves without dark matter.

The organizers and the participants made this an exciting and successful conference. The meet- 
ing was so superbly organized that not even a heavy rain shower was able to spoil the enjoyment of an authentic Swedish smorgasbord while boating through the idyllic Stockholm archipelago.

\section{References}

[1] Hinshaw et al. [WMAP Collaboration],

[2] M. Tegmark et al. [SDSS Collaboration], Astrophys. J. 606, 702 (2004) [arXiv:astro-ph/0310725].

[3] M. Tegmark et al. [SDSS Collaboration], Phys. Rev. D 74, 123507 (2006) [arXiv:astro-ph/0608632]. 